\title{
Electromagnetic Rod in Lower Limb Lengthening: A Technical Note for Shaft Osteotomy
}

\author{
Miguel Lopes ${ }^{1}$, Bernardo Nunes ${ }^{2}$, André Couto $^{3}$, Joana Freitas ${ }^{4}$, Rui Martins ${ }^{5}$, Jorge Coutinho ${ }^{6}$, Gilberto Costa $^{7}$
}

\begin{abstract}
Purpose: Long bone lengthening procedures are paramount in the treatment of limb length discrepancies. We witnessed a revolution in the treatment paradigm of this pathology with the development of expandable intramedullary rods. Endomedular nailing might be technically demanding and some steps are critical for success. The aim of authors is to describe a technical note of the PRECICE system that may ease femoral nailing and fixation: the nail can be advanced through the femur and proximal and distal fixation performed previous to complete femoral osteotomy.

Materials and methods: The authors present a case series of XX patients in which the limb lengthening has been performed with partial osteotomy with Gigli saw, nail advancement, proximal and distal fixation, and osteotomy completion at the end of the procedure.

Results: After 18 consecutive limb (femoral) lengthening operations with this technical variation in PRECICE, nail patients presented no significant lower limb length discrepancy. There were no records of rotational deformities, nonunion, or infection. Material failure was not reported.

Conclusion: This technical note is another positive variable that can help to ease the procedure, minimise possible complications, and confirm magnetic expandable nails as the gold standard technique in limb lengthening procedures and it might be applied to other nailing systems for limb lengthening procedures.

Keywords: Lengthening nail, Limb deformity, Limb lengthening.

Strategies in Trauma and Limb Reconstruction (2019): 10.5005/jp-journals-10080-1444
\end{abstract}

\section{INTRODUCTION}

Bone lengthening procedures are paramount in the treatment of limb length discrepancies. We witnessed a revolution in the treatment paradigm of this pathology with the development of expandable intramedullary nails, which are controlled electromagnetically allowing for continuous distraction in ambulatory setting with a single device, avoiding multiple procedures for lengthening adjustments.

The reported complication rate is generally low, the most common being joint contracture followed by implant failure to lengthen, nail breakage, and premature consolidation. ${ }^{1-4}$ Axial and rotational misalignments have also been described. ${ }^{5}$

One of the most important steps in the surgical technique is related to the osteotomy of the long bone. Difficulties in achieving alignment for nail passage and restoring anatomic axial and rotational alignment leading to inadequate rotation and limb dysfunction have been reported. ${ }^{5,6}$

The primary purpose of this study is to report a modification of femoral osteotomy in intramedullary lengthening nail and its results in the treatment of 18 patients with limb length discrepancy. This modification in femoral osteotomy may be helpful in preventing rotational misalignment and make the surgical procedure less prone to error.

\section{TeChNique}

Implant selection, entry point, reaming, osteotomy level, and fixation were performed according to surgical technique. The selected osteotomy site was marked and a $5-\mathrm{cm}$ skin incision performed. Dissection is carried to the bone.

A cable passer is used in order to protect soft tissues while placing the Gigli saw around the femur (Fig. 1). After this step,
${ }^{1-7}$ Department of Orthopaedic and Traumatology, Centro Hospitalar de São João, Porto, Portugal

Corresponding Author: Miguel Lopes, Department of Orthopaedic and Traumatology, Centro Hospitalar de São João, Porto, Portugal, Phone: +351 916981796, e-mail: jmiguelmlopes@gmail.com

How to cite this article: Lopes M, Nunes B, Couto A, et al. Electromagnetic Rod in Lower Limb Lengthening: A Technical Note for Shaft Osteotomy. Strategies Trauma Limb Reconstr 2019;14(3):139-141.

Source of support: Nil

Conflict of interest: None

incomplete osteotomy is performed leaving the lateral cortex intact (Fig. 2), so it can proceed with reaming, nailing, proximal, and distal fixation (Fig. 3). After these steps, osteotomy is completed (Fig. 4).

The standard postoperative protocol that includes pain control, early mobilisation, and protected weight bearing after 24 hours was applied. Wound dressings are changed two times per week and suture stitches removed after 12-14 days.

The lengthening protocol was initiated 7-10 days after osteotomy and nailing for patients younger than 10 years old, and approximately 14 days for patients older than 10 years. Daily lengthening is $0.75-1 \mathrm{~mm}$ divided into three sessions.

Weekly clinical and radiographic evaluations were performed to review the patient's progression.

\section{Clinical Experience}

From 2016 to 2019, 18 patients ( 15 female and 3 male) with mean age of 16 years (min. 11 years; max. 18 years) were admitted to our children's orthopaedic department with a diagnosis of limb length discrepancy and with clinical indication for the femoral lengthening 


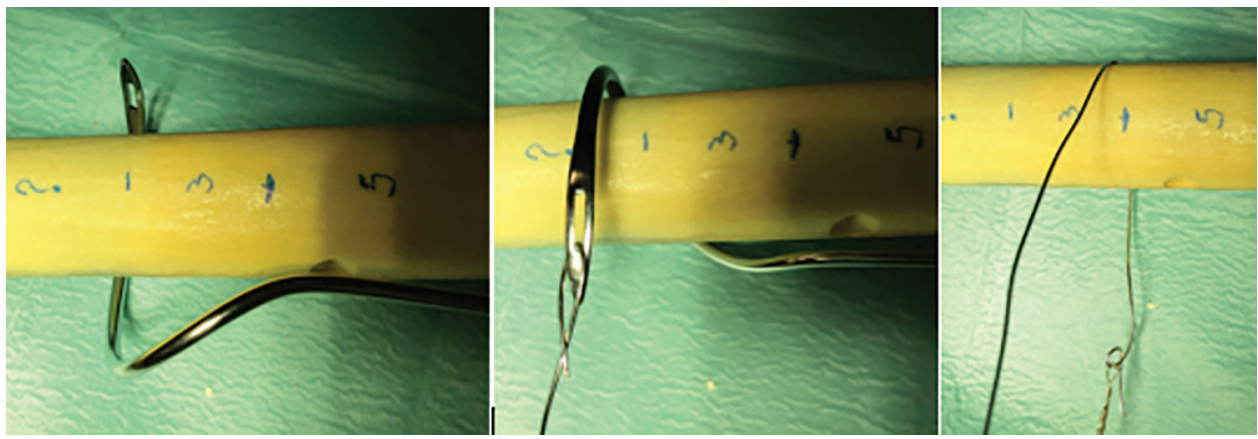

Fig. 1: Cable passer with steel wire used to pass Gigli saw around femur

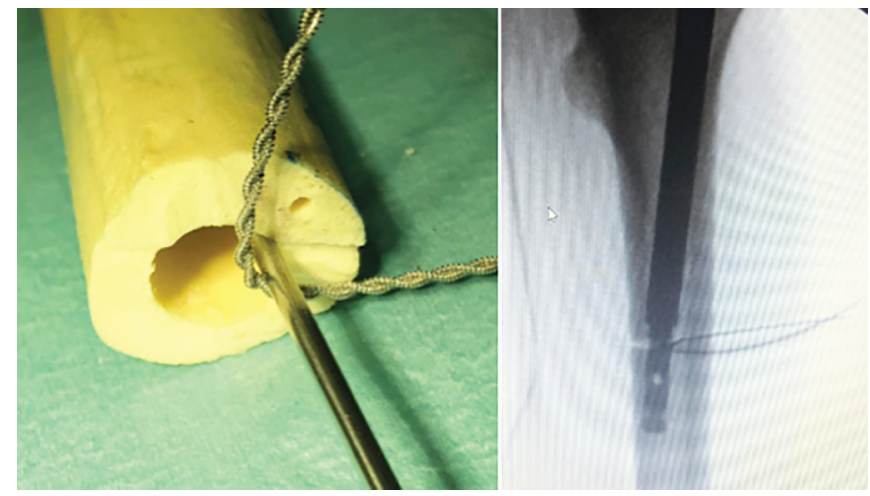

Fig. 2: Incomplete osteotomy. Note that due to small diameter of Gigli saw, there is enough room to nail passage through medullary canal

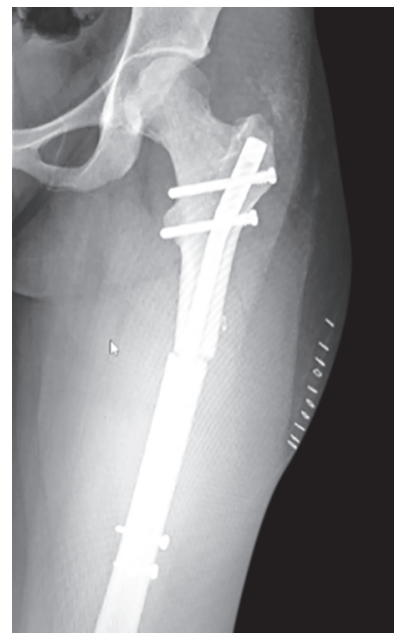

Fig. 4: Postoperative X-ray

procedure. Mean limb length discrepancy was $4.5 \mathrm{~cm}(\min .2 .5 \mathrm{~cm}$; $\max .6 \mathrm{~cm}$ ).

Femoral lengthening procedures were performed with the intramedullary magnetic nail_PRECICE (Nuvasive, California)— with the reported technical modification in osteotomy.

The standard postoperative and lengthening protocol was applied.

Mean follow-up time was 2.9 years (min. 1 year; max. 4 years). The mean bone healing index (BHI) was 41 days/cm (min. 35 days/ $\mathrm{cm}$; max. 49 days/cm). After the completion of lengthening, mean limb length discrepancy was $0.5 \mathrm{~cm}$ ( $\min .0 \mathrm{~cm}$; max. $1.5 \mathrm{~cm}$ ).

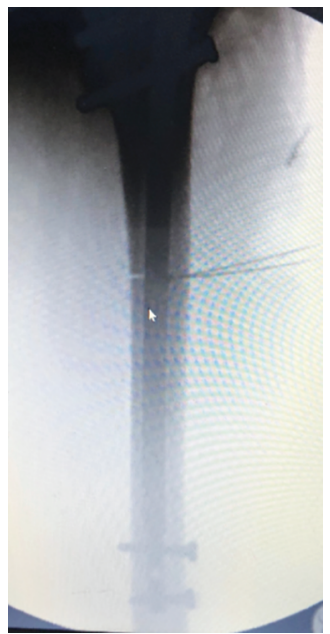

Fig. 3: Femoral nailing with proximal and distal locking screws before completing osteotomy

There were no records of rotational deformities, nonunion, or infection. One case of mechanical failure was reported 3 weeks after surgery due to noncompliance with recommendation of protected weight bearing.

Bone union was recorded through X-ray assessment from a senior orthopaedic surgeon once three out of four cortices have consolidated. After documented bone union, the patient is advanced to weight bearing as tolerated until full weight bearing.

\section{Discussion}

One aspect that becomes clear when reviewing the available literature addressing limb lengthening is that over the past few years we have witnessed extraordinary advances in surgical techniques to address this pathology. Since first descriptions of the Ilizarov method, passing to sophisticated circular external fixators and software programs to gradually correct complex deformity (Hexapod) and evolving to the introduction of magnetic expandable intramedullary nails. ${ }^{7-9}$

The aim of this paper is to describe a modification in the PRECICE nail surgical technique in order to avoid rotational deformity.

Most lengthening surgeons perform a prescriptive osteotomy that is a recipe for success: percutaneous drill holes, closed reaming that deposits the reamings at the osteotomy site, and then percutaneous femoral osteotomy with an osteotome. For this method, Laubscher et al. described femoral BHI of 31.3 days $/ \mathrm{cm}^{10}$ 
The authors present an mini-open osteotomy that is completed after femoral nailing and nail fixation. This modification has the ability to fix the nail proximally and distally prior to completing the osteotomy to ensure no change in rotation.

With the mini-open osteotomy, BHI was 41 days/cm (min. 35 days $/ \mathrm{cm}$; max. 49 days $/ \mathrm{cm}$ ). As bone from reaming still is deposited at the osteotomy site, this difference may be explained by the additional surgical dissection needed.

Although $\mathrm{BHI}$ was superior, we did not identify major complications caused by this modification and all patients went to bone consolidation. This technique has the advantage to facilitate nail advance and assuring no rotational deformities. We call attention for the fact that it does not allow for deformity correction and is only applicable for straight bones. This procedure does not address axis deviation due to limb lengthening per se as described by Burghardt et al. ${ }^{5}$

To our knowledge, there are no studies published relative to immediate postoperative deformity due to technical difficulties in femoral nailing and osteotomy.

We believe this series of cases is the first being described in which femoral lengthening has been performed with the PRECICE nail and in which osteotomy was completed after femoral nailing and nail fixation. Notwithstanding the use of PRECICE nail, this technical note might be applied to other nailing systems for limb lengthening procedures in order to ease the procedure and minimise the possibility of rotational deformity.

\section{CONCLUSION}

Osteotomy completion after femoral nailing and nail fixation is a technical tip useful to prevent rotational misalignment when using intramedullary lengthening nails. This technical note is another positive variable that can help to ease the procedure, minimise possible complications, and confirm magnetic expandable nails as the gold standard technique in limb lengthening procedures and it might be applied to other nailing systems for limb lengthening procedures.

\section{Ethical Approval}

All procedures performed in the study involving human participant were in accordance with the ethical standards of the institutional committee and with the 1964 Helsinki declaration and its later amendments or comparable ethical standards.

\section{Consent}

A written patient consent and permission to publish have been obtained. The images are completely anonymous (radiographs) and the text do not carry any identifying information.

\section{References}

1. Calder PR, Laubscher M, Goodier WD. The role of the intramedullary implant in limb lengthening. Injury 2017;48(Suppl 1):S52-S58. DOI: 10.1016/j.injury.2017.04.028.

2. Kirane YM, Fragomen AT, Rozbruch SR. Precision of the PRECICE internal bone lengthening nail. Clin Orthop 2014;472(12):3869. DOI: 10.1007/s11999-014-3575-0.

3. Paley D, Harris M, Debiparshad K, et al. Limb lengthening by implantable lengthening devices. Tech Orthop 2014;29(2):72. DOI: 10.1097/BTO.0000000000000072.

4. Schiedel FM, Vogt B, Tretow $\mathrm{HL}$, et al. How precise is the PRECICE compared to the ISKD in intra-medullary limb lengthening? Acta Orthop 2014;85(3):293. DOI: 10.3109/17453674.2014.913955.

5. Burghardt RD, Paley D, Specht SC, et al. The effect on mechanical axis deviation of femoral lengthening with an intramedullary telescopic nail. J Bone Joint Surg Br 2012;94(9):1241-1245. DOI: 10.1302/0301620X.94B9.28672.

6. Muthusamy S, Rozbruch SR, Fragomen AT. The use of blocking screws with internal lengthening nail and reverse rule of thumb for blocking screws in limb lengthening and deformity correction surgery. Strategies Trauma Limb Reconstruction 2016;11(3):199-205. DOI: 10.1007/s11751-016-0265-3.

7. Birch J. A brief history of limb lengthening. J Pediatr Orthop 2017;37(Suppl 2):S1-S8. DOI: 10.1097/BPO.0000000000001021.

8. Calder P, McGrath A, Chasseaud M, et al. The PRECICE intramedullary limb lengthening system. Bone Joint J 2013;95-B(Suppl. 23):11.

9. Xu WG. Comparison of intramedullary nail versus conventional llizarov method for lower limb lengthening: a systematic review and meta analysis. Orthop Surg 2017;9(2):159-166. DOI: 10.1111/ os.12330.

10. Laubscher M, Mitchell C, Timms A, et al. Outcomes following femoral lengthening: an initial comparison of the PRECICE intramedullary lengthening nail and the LRS external fixator monorail system. Bone Joint J 2016;98-B(10):1382-1388. DOI: 10.1302/0301620X.98B10.36643. 